\title{
Perceção de experiências marcantes do confinamento em famílias portuguesas e brasileiras: Um estudo qualitativo
}

\author{
Laura Magalhães ${ }^{1}$, Célia Nascimento ${ }^{2}$, Ana P. Antunes ${ }^{3}$, Silvana \\ Martins $^{4}$, Maria Angela Mattar Yunes ${ }^{5}$, Ana Almeida ${ }^{1}$
}

${ }^{1}$ Centro de Investigação em Estudos da Criança, Universidade do Minho, Portugal | monteiro.laurapatricia@gmail.com; aalmeida@ie.uminho.pt | https://orcid.org/0000-0002-15419301; https://orcid.org/0000-0003-0036-312X

2 Departamento de Psicologia Social e do Desenvolvimento, Universidade Federal do Espírito Santo, Brasil |celia.nascimento@ufes.br | https://orcid.org/0000-0002-3175-0715

${ }^{3}$ Departamento de Psicologia, Universidade da Madeira, Centro de Investigação em Estudos da Criança, Universidade do Minho Portugal | aantunes@uma.pt | https://orcid.org/0000-0002-33367867

${ }^{4}$ Unidade de Investigação em Ciências da Saúde: Enfermagem, Escola Superior de Enfermagem de Coimbra, Portugal | b12012@ese.uminho.pt | https://orcid.org/0000-0003-3791-3236

5 Programa de Pós-Graduação em Psicologia, Universidade Salgado de Oliveira/Universo, Niterói,

RJ, Brasil | mamyunes@gmail.com | https://orcid.org/0000-0002-4653-3895

Resumo: A atual situação pandémica da COVID-19 impôs condições de confinamento que alteraram as rotinas das famílias, suscitando ajustes das práticas parentais. Neste contexto de ajustamento entre tensão e apoio, o presente estudo teve por objetivo analisar a perceção de experiências marcantes do confinamento de famílias em Portugal e no Brasil. Como parte de uma pesquisa mais ampla, foi aplicado um questionário online, com questões de caracterização sociodemográfica e uma pergunta aberta focando os acontecimentos marcantes da vida em família no período do confinamento. Foram analisadas as respostas de 280 pais e mães em Portugal e 94 no Brasil recorrendo ao modelo da teoria fundamentada nos dados. Na codificação das respostas prosseguiu-se sistematicamente de uma análise inicial aberta para a categorização de eixos temáticos. Múltiplos passos foram seguidos para a validação do estudo, com auxílio do software QSR N'Vivo, versão 12 para Mac. Verificouse nos dois países, mesmo considerando as diferenças no tempo de confinamento e as diferenças culturais, que a vivência do confinamento trouxe ganhos e dificuldades para as famílias. As dificuldades referem-se às mudanças na organização familiar e efeitos na saúde física e mental. No tocante aos ganhos, o confinamento trouxe novos significados às dinâmicas familiares, salientando-se a melhoria no relacionamento com os filhos e entre 0 casal. Foram reportadas ainda repercussões positivas na vida pessoal. Os resultados indicam que os aspectos marcantes no enfrentamento dos desafios causados pelo confinamento sugerem a necessidade de apoio à parentalidade e promoção de resiliência familiar e parental.

Palavras-chave: Família; Parentalidade; Resiliência; Confinamento; Covid-19.

Perception of Impressive Experiences During the Confinement on Portuguese and Brazilian Families: A Qualitative Study

Abstract. The current pandemic situation due to COVID-19 has constrained confinement measures, which changed family routines, leading to adjustments of parental practices. In this context of balance between tension and support, the present study aimed at analyzing the perception of impressive experiences during the confinement in family life in Portugal and Brazil. As part of a broader research, it was applied an online questionnaire with questions about sociodemographic data and an open question focusing on important events in the family life during the lockdown period. Answers to the question were analyzed from 280 fathers and mothers in Portugal and 94 in Brazil using the grounded theory principles. The coding of the collected responses proceded systematically from an initial open to axial categories, aided by the QSR N'Vivo software, version 12 for Mac. Multiple steps were used to the trustworthiness of the study. It was found, in both countries, despite the differences in confinement time between respondents and cultural differences, that the confinement experience brought difficulties and gains for the Brazilian and the Portuguese families. The difficulties were referred to changes in family organization and consequences on the physical and mental health of its members. Related to gains, confinement allowed a greater coexistence and the re-signification of family dynamics, with gains being identified in proximal relationships with the children, between the couple, and among family members in general. Positive repercussions on personal life were also reported. The results indicate the need of parenting support and strengthening of family and parental resilience.

Keywords: Family; Parenting; Resilience; Lockdown; COVID-19. 


\section{Introdução}

Em março de 2020, após rápida escalada da COVID-19, a Organização Mundial de Saúde (2020) declarou esta doença como sendo uma pandemia global. Neste contexto, foram adotadas medidas restritivas para prevenir e controlar os contágios. Entre as várias medidas sanitárias que começaram a ser discutidas e aplicadas, o confinamento social foi uma delas. Esta realidade afetou a vida das famílias, restringindo a circulação de pessoas e interrompendo convívios sociais. Prevaleceu o teletrabalho e as atividades escolares em casa.

Tanto em Portugal como no Brasil, países onde se realizou o presente estudo, é reconhecido que o confinamento social alterou as práticas parentais, suscitando ajustes nas atividades quotidianas e novas rotinas. Sem o apoio direto e a presença física da rede de apoio, restringem-se as relações, há uma sobrecarga de atividades no contexto doméstico e intensifica-se a convivência entre os elementos do mesmo agregado familiar.

No quadro dos estudos da parentalidade, estas necessidades de ajustamento são a base para investigar a resposta de mães e pais e a percepção do seu papel para a continuidade do funcionamento familiar e do bem-estar da criança. Por sua vez, é reconhecido que os fatores contextuais exercem uma influência determinante no exercício da parentalidade $e$ que o equilíbrio dos fatores de stress e de suporte contribui, como contraponto à promoção do bom trato, para a prevenção dos maus tratos às crianças (Belsky, 1984). Os cuidados parentais associados às manifestações de carinho, atenção e sensibilidade, aos estímulos e incentivos à autonomia, à flexibilidade para adaptar ou compensar a falta das fontes de apoio habituais dos filhos constituíram-se em novos recursos e capacidades de pais, reforçando a sua parentalidade e a resiliência parental. Associado ao conceito de parentalidade encontra-se o conceito de resiliência que, segundo Barudy e Dantagnan (2010) diz respeito a:

"capacidades dos cuidadores principais para fornecer às crianças e adolescentes: cuidados que promovam o desenvolvimento positivo, modelos e fontes de apoio para superação das adversidades, práticas e valores que proporcionam confiança pessoal e no ambiente para passar pelos desafios da vida" (p.248).

Para esses mesmos autores, a articulação entre recursos ambientais e sociais, o próprio sistema familiar e os recursos pessoais são fontes cruciais para a resiliência parental (Barudy \& Dantagnan, 2010). A propósito da resiliência parental Rodrigo (2009) retrata-a como uma parte sensível do sistema familiar, a qual pode ser afetada, sobretudo, nas famílias que vivenciam situações adversas e de stress psicossocial. Todavia, mesmo em situações de tensão, os adultos podem desenvolver mecanismos que os tornam capazes de exercitar a sua parentalidade com sucesso (Rodrigo et al., 2015). Potencialmente, algumas respostas às alterações levam à descoberta de aspetos benéficos à comunicação pais-filhos, ao acompanhamento das atividades, ao conhecimento de hábitos, capacidades, mas também de preocupações, receios e dificuldades.

Alguns estudos já foram sendo realizados e os resultados apontam para efeitos em diferentes domínios de forma diferenciada. Assim, por exemplo, no Brasil, no estudo de Silva, Schmidt, Lordello, Noal, Crepaldi, \& Wagner(2020), os investigadores destacam os efeitos ao nível da dinâmica da relação do casal e da necessidade de divisão de tarefas mais igualitárias. Num outro trabalho, os resultados destacam o agravamento das desigualdades socioeconómicas (Silva, Lordello, Schmidt, \& Mietto, 2020).

Em Portugal, num estudo do Instituto de apoio à Criança (2020) destaca-se a dificuldade de gerir o excesso de tarefas escolares que, para muitas crianças e pais, se revelou uma sobrecarga importante no tempo de confinamento. Finalmente, o estudo de Almeida et al. (2020), salienta que o tempo em confinamento reclamou maior conciliação entre trabalho e a família, diminuiu o tempo pessoal e para o casal; tendo, contudo, a consciência destas exigências influenciado positivamente a perceção de competência dos pais para controlar o stress e atender às solicitações dos filhos. 
É precisamente no âmbito deste último estudo que se enquadra o presente trabalho. $\mathrm{Na}$ continuação do estudo acerca das mudanças na parentalidade em tempos de COVID-19, o foco do presente trabalho incide nas experiências que as mães e pais pontuaram como tendo maior impacto durante o confinamento, compreendendo os acontecimentos que os influenciaram a nível pessoal e familiar. A inclusão desta questão aberta teve por objetivo obter uma informação espontânea acerca de acontecimentos com especial valor emocional e a percepção das mães e dos pais das experiências vividas no tempo do confinamento. A análise destas respostas permite aprofundar o conhecimento sobre 0 fenómeno em estudo, nomeadamente, o relacionado com a pandemia atual (Teti et a., 2020). Neste quadro pandémico, o presente estudo teve por objetivo analisar a perceção de experiências marcantes do confinamento social na vivência das famílias em Portugal e no Brasil, a partir de uma metodologia de análise qualitativa.

\section{Método}

\subsection{Caracterização dos Participantes}

A amostra portuguesa é constituída por 280 participantes (92.1\% do sexo feminino), predominantemente de nacionalidade portuguesa $(94.6 \%)$ e com idades compreendidas entre os 22 e os 58 anos ( $M=40.06 ; D P=6.45)$. Estas famílias, em média, ficaram 58,49 dias em confinamento.

No que diz respeito à amostra brasileira, a mesma é constituída por 94 participantes (88.3\% do sexo feminino), maioritariamente de nacionalidade brasileira $(97.9 \%)$ e com idades compreendidas entre os 25 e os 58 anos $(M=42.41 ; D P=6.80)$. Em média, estas famílias ficaram 119,03 dias em confinamento.

A amostra foi de conveniência e participaram do estudo pais e mães, ou pessoas que desempenhavam esse papel junto das crianças e adolescentes, em famílias de qualquer configuração, com pelo menos um filho com idade inferior a 18 anos, residindo à data na mesma habitação.

\subsection{Procedimento de Recollha de Dados}

A recolha de dados foi feita a partir de divulgação de um questionário estruturado de autoaplicação, que foi preenchido pelos participantes em plataforma online, e divulgado nas redes sociais.

Em Portugal, os dados foram recolhidos entre maio e junho de 2020, e no Brasil entre agosto e novembro de 2020. Entre outras questões, o questionário foi composto pela seguinte pergunta aberta, na qual nos centramos no presente estudo: Gostaria de referir alguma situação que tenha marcado o tempo de confinamento em família?

Em Portugal, o estudo foi aprovado pela Comissão de Ética para Investigação em Ciências Sociais e Humanas da Universidade do Minho CEICSH045/2020, no dia 13 de maio de 2020. No Brasil, o projeto foi aprovado pelo Comitê de Ética do Brasil (CONEP), sob o parecer n. -4.157 .400 . Em termos das Considerações Éticas, todos os participantes foram informados, antes de responderem ao questionário, sobre os objetivos e métodos da pesquisa, a voluntariedade na participação e a confidencialidade dos dados.

\subsection{Procedimentos de Análise dos Dados}

A análise das respostas à questão aberta do questionário, foi realizada através da análise de conteúdo, conforme proposto por Bardin (1977) descrito por Mendes e Minkulin (2017). 
Seguindo os passos do método de análise, realizou-se inicialmente uma pré-análise dos dados, através de uma leitura flutuante das respostas e a elaboração do sistema de categorias de forma indutiva e independente. Posteriormente, a equipa reuniu para análise e discussão, realizando assim uma revisão e modificação do sistema inicial de categorias, tendo por base a literatura consultada e os objetivos do estudo. A equipa de investigadores dos dois países que realizaram a categorização dos dados foi constituída por pelo menos dois pesquisadores de cada país. A organização e análise dos dados ocorreu com o auxílio ao software QSR N'Vivo, versão 12 para Mac. As equipas reuniram virtualmente para reflexão e análise conjunta dos dados.

$\mathrm{Na}$ exploração inicial do material, entre as experiências marcantes identificadas por mães e pais relativas ao período do confinamento, verificou-se que os conteúdos registados revelaram percepções que implicaram ganhos para os participantes no estudo e/ou para as suas famílias, bem como, dificuldades sentidas pelos mesmos. Quando um mesmo participante descreveu a sua perceção, relatando dois aspectos, os trechos das respostas foram separados para compor unidades de registos inseridas separadamente na organização dos códigos. Após essa organização de conteúdos, procurou-se novos agrupamentos com a verificação das semelhanças e diferenças em relação ao conteúdo das respostas, posterior codificação e categorização em função dos temas que se destacaram.

Com o aprofundamento da análise, os conteúdos que registaram ganhos e dificuldades durante o confinamento foram subdivididos em eixos temáticos definidos a partir dos domínios em que se exercia a parentalidade (filhos, família, casal, vida pessoal e comunidade escolar). $\mathrm{Na}$ amostra portuguesa constatou-se o surgimento da dimensão da relação com a comunidade escolar, que não foi valorizada pela amostra brasileira.

Após essa subdivisão, houve nova exploração das unidades de registos para organização de outra etapa de categorização em cada eixo temático.

Assim, uma vez que a pergunta aberta conduzia os participantes a descreverem experiências marcantes e a perceção das mesmas no contexto familiar, os autores deste estudo focaram-se na "perceção pessoal" e nos "domínios relacionais" que emergiram. Depois de uma leitura inicial e da discussão, três avaliadores caracterizaram a perceção pessoal das experiências em função dos ganhos e dificuldades nos diferentes domínios relacionais (relação com os filhos, na relação com a família, relação do casal, na vida pessoal e a relação com a comunidade escolar).

Adicionalmente, foram analisadas as áreas de conteúdos como sendo específicas a cada um dos países, contemplando esses temas no seu conjunto e potenciais diferenças associadas às políticas de confinamento, às características das próprias famílias e outras questões culturais.

\section{Resultados}

\subsection{Organização das Respostas em Portugal e no Brasil}

A Tabela 1 apresenta a síntese dos resultados da amostra dos dois países.

Tabela 1. Aspectos marcantes para as famílias do confinamento.

\begin{tabular}{lllcc}
\hline Dimensões & Categorias & Subcategorias & $\begin{array}{c}\text { Portugal } \\
\%\end{array}$ & $\begin{array}{c}\text { Brasil } \\
\%\end{array}$ \\
\hline \multirow{2}{*}{ Vida pessoal } & \multirow{2}{*}{ Ganhos } & Gravidez ou nascimento de um filho & 2.5 & 0.00 \\
& & Mudança de hábitos e aprender coisas novas & 5.71 & 6.38 \\
& \multirow{2}{*}{ Dificuldades } & $\begin{array}{l}\text { Falta de tempo e de espaço individual } \\
\text { (necessidade de estar sozinho) }\end{array}$ & 1.43 & 0.00
\end{tabular}


Vol. 9 | Investigação Qualitativa em Ciências Sociais: Avanços e Desafios

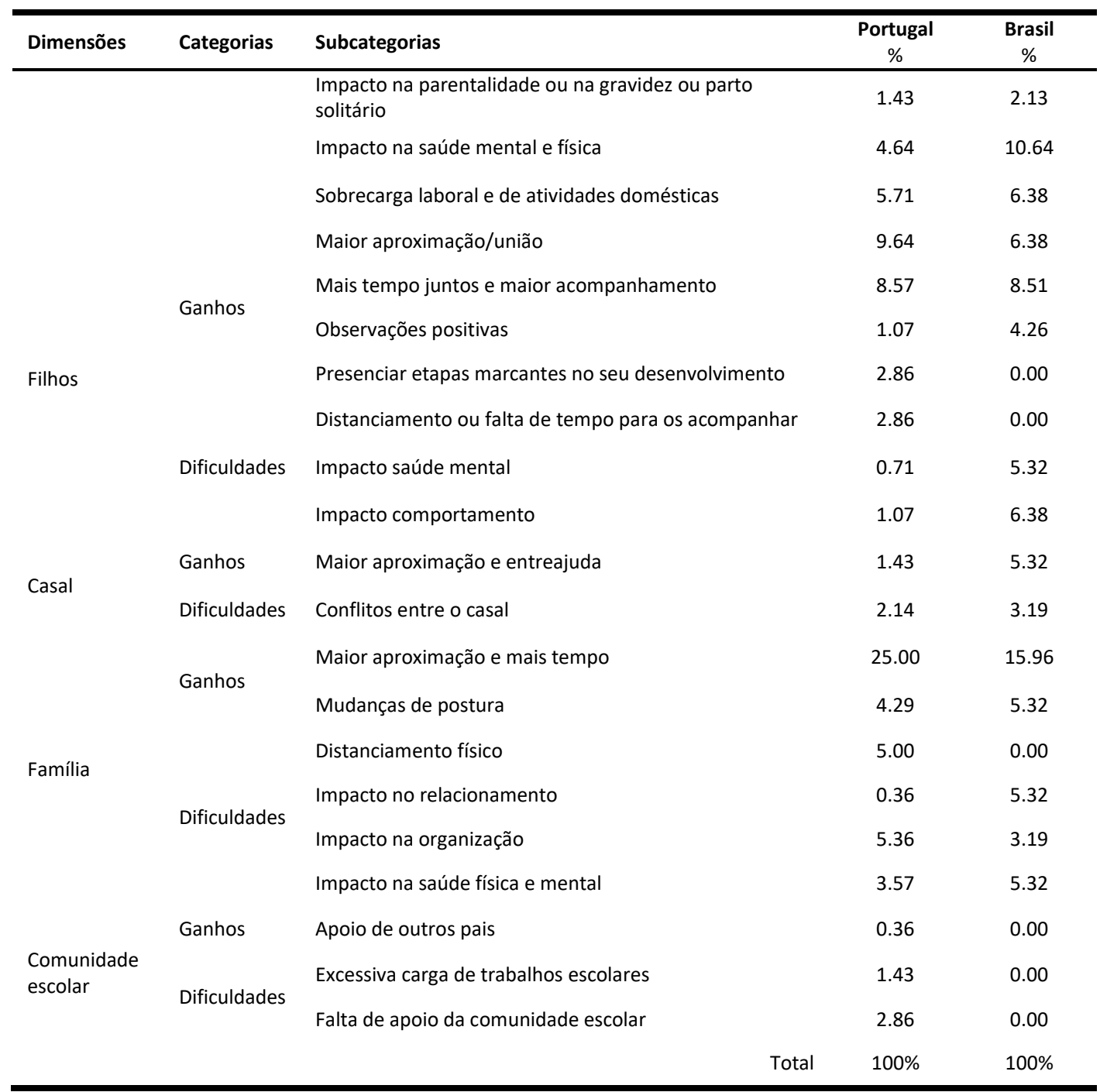

O confinamento proporcionou uma maior convivência e novos significados à parentalidade que envolveram ganhos para as relações com os filhos e para a família de forma geral, como a possibilidade de ter mais tempo juntos e uma maior aproximação. Os participantes, na sua maioria mães, valorizaram a possibilidade de passarem mais tempo em casa com os filhos, e com isso acompanhar o seu desenvolvimento e realizarem atividades em conjunto. Dinâmicas que anteriormente eram dificultadas pela rotina diária do contexto laboral são valorizadas nos relatos: "Poder passar um tempo com meu filho e acompanhar seu desenvolvimento, que não aconteceria de outra forma" (participante brasileiro). Do mesmo modo, um participante português refere "Acho que foi um momento de desfrutar da casa com a familia toda. Mais atividades juntos, jogos de tabuleiro e visionamento de filmes".

Foram ainda relatados ganhos na vida pessoal dos participantes que avaliaram o confinamento como uma oportunidade para mudanças de hábitos e a possibilidade de aprendizagens geradas pelo quotidiano do período de confinamento social, tais como: fazer pão, cuidar da decoração e fazer as tarefas com mais calma. Como demonstrado no relato a seguir: "Maior investimento em coisas para casa, plantas, decoração..." (participante brasileiro). Importa ainda referir que, na amostra portuguesa, a gravidez ou o nascimento de um novo filho foi um dos acontecimentos marcantes e valorizado em termos pessoais, como descrito: "Este confinamento fica marcado por estar a passar a $3^{a}$ gravidez de forma especial" (participante português). 
Para a relação do casal, apesar de os relatos de conflitos e divórcio terem sido registados nos dois países, importa referir que para a amostra brasileira o confinamento proporcionou oportunidade para mais união e parceria na divisão de tarefas, como mostra o registro: "Marcante a parceria com o marido nas atividades domésticas". Já na amostra portuguesa, os onflitos entre o casal apareceram mais vezes mencionados. Por outro lado, os relatos reforçam o confinamento como uma oportunidade para estreitar laços familiares com a convivência mais estreita entre os cuidadores e os seus filhos, assim como entre irmãos, além de mudanças de postura em família que contribuíram para momentos de maior tolerância, apoio mútuo, adoção de momentos de lazer em conjunto e adesão a práticas de espiritualidade, conforme relatos: "Vejo que estamos mais unidos e confiantes em Deus" (participante brasileiro); "Rezar o terço em família" (participante português) ou "Maior cumplicidade entre os membros da família" (participante português).

Entre as dificuldades do período de confinamento verificou-se que, também nos dois países, destacam-se respostas que demonstram o impacto do isolamento social para a saúde, para os relacionamentos e para aspectos que envolvem a organização das famílias. Foram mencionados impactos na saúde física, mental e no comportamento dos filhos, tanto da família como dos próprios participantes. Respostas sobre crises de ansiedade e choro dos filhos e do participante, exaustão emocional na família, vivência de perda e luto solitário, entre outras situações, revelam o impacto do confinamento para os membros da família, no que à saúde emocional diz respeito, como no exemplo: "Crises de choro de meu filho devido ao confinamento têm sido preocupantes" (participante brasileiro). Doenças na família e o enfrentamento do Covid-19 também foram valorizados pelos participantes nos dois países.

Em relação às dificuldades, se para os participantes brasileiros o impacto do comportamento dos filhos foi o mais valorizado, para os participantes portugueses, o distanciamento dos filhos ou a falta de tempo de qualidade para os acompanhar foi 0 aspeto mais vezes mencionado.

Nos relacionamentos na família, os participantes portugueses mencionaram o distanciamento imposto pelo confinamento da família alargada e dos amigos, como sendo o aspeto mais marcante. Já para os participantes brasileiros, o impacto nos relacionamentos e na saúde física e mental na família foram os aspetos mais vezes mencionados. Para os participantes dos dois países, a organização da família também foi afetada com o confinamento, como a falta de controlo da rotina familiar e impacto na vida financeira.

Entre as dificuldades para a vida pessoal, nos dois países, destacam-se o impacto na saúde física e mental e a sobrecarga de trabalho e atividades domésticas, mencionadas principalmente pelas mães, como descrito: "Dificuldade de conciliar teletrabalho, apoio aos filhos e trabalho doméstico" (participante português); "Esgotamento físico e mental para dar conta das atividades domésticas, profissionais e acadêmicas" (participante brasileiro).

A relação com a comunidade escolar, nomeadamente as dificuldades sentidas, foi uma dimensão mencionada de forma exclusiva pelos participantes portugueses. Estes consideraram haver uma sobrecarga excessiva de trabalhos escolares, bem como, uma falta de apoio da comunidade escolar para com as famílias: "Pouca adaptabilidade das escolas para acolher dificuldades das famílias" (participante português).

\section{Discussão e Conclusões}

O início da aplicação dos questionários em relação ao tempo de confinamento foi diferente nos dois países: em Portugal a aplicação do questionário iniciou-se 2 meses após o período de confinamento e no Brasil aconteceu após 4,5 meses. Embora esse tempo possa ter configurado vivências diferentes para as famílias nos dois países, constatou-se, a partir da análise de conteúdo, que o confinamento trouxe dificuldades e ganhos, com pontos em comum, para as famílias portuguesas e brasileiras. 
Ao descreverem aspectos marcantes para a família durante o confinamento, pais e mães portuguesas e brasileiras descreveram situações envolvendo dificuldades provocadas por esse evento, bem como, situações que exigiram estratégias de enfrentamento, flexibilidade e adaptação às mudanças no âmbito de uma nova dinâmica familiar e relacional vivenciadas a partir deste.

A parentalidade é uma função relacional, que envolve um vínculo afetivo e de cuidado com os filhos, para que estes tenham uma base segura para se desenvolverem. Contudo, no exercício dessa função é importante que a família tenha uma rede de apoio. Relações familiares, comunitárias e institucionais que auxiliem o exercício da parentalidade são importantes (Rodrigo et al., 2015), especialmente quando as condições de vida trazem desafios que concorrem com o cuidado e a proteção no ambiente familiar, como no caso da pandemia e do confinamento.

O confinamento dificultou o acesso a outros espaços e à rede de apoio familiar, conforme descrito pelos participantes portugueses. Sabe-se que a rede de apoio pessoal é um importante elemento de proteção em situações adversas (Masten, 2014). Portanto, na ausência destes recursos foi referida a sobrecarga laboral e das atividades domésticas com impacto na saúde mental dos cuidadores e dificuldades de acompanhamento dos filhos, conforme demonstrado nos resultados. Esses dados são coerentes com resultados e análises discutidos em outros estudos (Coyne et al., 2020; Silva, Schmidt et al., 2020).

Numa perspetiva macrossistémica, o cenário da pandemia causada pelo SARS-coV-2 e o isolamento social como medida de proteção ao contágio, provocaram mudanças em diferentes espaços de convivência social. Especialmente no contexto da família, isso tem afetado as rotinas, as relações e a a saúde dos seus membros (Silva, Schmidt et al., 2020).

Situações de conflitos na família, envolvendo pais e filhos, irmãos e a relação do casal também foram registadas pelos participantes de ambos os países. No estudo de Silva, Schmidt et al. (2020), o confinamento configura um desafio para os casais Brasileiros, por colocar a relação e a saúde dos cônjuges em situação de maior vulnerabilidade. Os cuidados com a saúde mental, os valores compartilhados entre os casais, os padrões de comunicação e a parceria na divisão de tarefas em família são fatores que influenciam a dinâmica do casal e seus recursos para enfrentarem os desafios da vida familiar e conjugal. Nos resultados encontrados, verificou-se que tanto relatos sobre oportunidade de estreitamento dos laços e maior parceria, quanto conflitos e rompimento foram vivenciados pelos casais durante o confinamento.

De acordo com os participantes, o confinamento trouxe também impacto para a organização familiar, envolvendo a flexibilidade nas rotinas e trazendo dificuldades financeiras. De facto, no Brasil, pesquisas mostram a expressiva redução no orçamento das famílias e a perda do emprego, em especial no caso de mulheres e pessoas com menores índices de escolaridade (Hecksher, 2020). No caso de Portugal, os estudos são escassos, no entanto, alguns trabalhos divulgados apontam alterações nas dinâmicas familiares (Almeida et al., 2020).

No entanto, pais e mães também consideraram que o confinamento trouxe vivências que foram interpretadas como ganhos. A maior aproximação com a família e entre o casal, e particularmente com os filhos, com a possibilidade de passar mais tempo com eles, fazendo atividades juntos e acompanhando o seu desenvolvimento são referências expressivas nos dois países. Essa avaliação dos cuidadores mostra que a família, mesmo em situações de stress e adversidade, pode encontrar recursos intrafamiliares e forças para enfrentarem as suas dificuldades. Weber e colaboradores (2006), destacam a expressão do afeto, a disponibilidade e o envolvimento dos pais na vida dos filhos e o acompanhamento como aspetos relevantes para a qualidade da relação familiar entre pais e filhos. A atenção emocional aos filhos e a capacidade de ser fonte de apoio em situações de stress e desafios são considerados aspectos importantes para a resiliência parental e para o desenvolvimento da resiliência nos filhos (Barudy \& Dantagnan, 2010; Rodrigo, 2009). 
Parece-nos que este estudo contribui para compreender como algumas experiências durante o confinamento foram sentidas como marcantes na vivência das famílias em Portugal e no Brasil. A resposta à pergunta aberta formulada permitiu perceber o alcance da implicação da COVID-19 ao nível familiar, filial, conjugal, pessoal e da comunidade escolar e a respetiva diferenciação de países. Parece-nos que este estudo ilustra a pertinência dos métodos qualitativos na compreensão do significado das experiências vividas e, como Teti et al. (2020) referem, na compreensão dos mecanismos sociais inerentes a esta pandemia, permitindo perceber também a complementariadade compreensiva dos dados qualitativos à leitura de dados quantitativos (Creswell, 2009). No entanto, podemos apontar algumas limitações ao estudo, nomeadamente, o número mais reduzido de participantes que optou por responder à questão aberta (facultativa e no final do questionário) em relação às outras questões, bem como o registo de um viés de participação superior das mães em relação aos pais e o tempo diferente de confinamento entre os dois países. Além disso, a própria recolha de dados online condicionou a participação no estudo, pois exigia alguma literacia digital.

\section{Agradecimentos}

Trabalho financiado por Fundos Nacionais através da FCT - Fundação para a Ciência e Tecnologia no âmbito do projeto do CIEC (Centro de Investigação em Estudos da Criança da Universidade do Minho) com referência UIDB/00317/2020

\section{Referências}

Almeida, A., Antunes, A., Martins, S., Nunes, C., Cruz, O., Oliveira, C., Pereira, D., Serrano, A., \& Sarmento, T. (outubro de 2020). Efeitos do confinamento na parentalidade em tempo de COVID-19. Comunicação por convite em simpósio no 8th International Congress of Educational Sciences and Development, realizado online (vídeoconferência).

Bardin, L. (1977). Análise de conteúdo. Lisboa: Edições 70.

Barudy, J. \& Dartagnan, M. (2010). Los Buenos tratos a la infancia. Parentalidad, apego y resiliencia. Barcelona: Gedisa.

Belsky, J. (1984). The determinants of parenting. Child Development, 55, 83-96. doi: https://doi.org/10.2307/1129836

Creswell, J. W. (2009). Research design: Qualitative, quantitative, and mixed methods approaches. Thousand Oaks California: Sage.

Coyne L., Gould E., Grimaldi M., Wilson K., Baffuto G., \& Biglan A. (2020). First things first: Parent psychological flexibility and self-compassion during COVID-19. Behavior Analysis in Practice, 1-7. doi:10.1007/s40617-020-00435-w.

Fundação Oswaldo Cruz. (2020). ConVid pesquisa de comportamentos. Retirado de https://convid.fiocruz.br/index.php?pag=principal.

Hecksher, M. (2020). Mercado de trabalho: A queda da segunda quinzena de março, aprofundada em abril. IPEA - Carta de Conjuntura, 47(2o Trimestre de 2020). Retirado de https://www.ipea.gov.br/portal/images/stories/PDFs/conjuntura/200619_cc47_nt.pdf

Instituto de apoio à Criança (2020). Estudo as crianças e jovens em isolamento social: síntese de resultados. Retirado de https://criancasatortoeadireitos.files.wordpress.com/2020/07/sintesede-resultados-as-crianc3a7as-e-jovens-em-isolamento-social.pdf

Masten, A. (2014). Ordinary Magic: Resilience Processes in Development. New York, London: The Guilford Press.

Mendes, R. \& Minkulin, R. (2017). A análise de conteúdo como uma metodologia. Cadernos de Pesquisa, 47(165), 1044-1066. https://doi.org/10.1590/198053143988.

Rodrigo, M. (2009). Una mirada integradora de la resiliencia parental: desde el contexto hasta la mente de las madres y los padres en riesgo psicosocial. Psicologia da Educação, 28, 51-71. 
Rodrigo, M., Máiquez, M., Martín, J., \& Rodriguez, B. (2015). La parentalidad positiva desde la prevención y promoción. In M. Rodrigo, M. Máiquez, J. Martin, S. Byrne, B. Rodríguez, Manual Práctico en Parentalidad Positiva (pp. 25-43). Madrid: Síntesis.

Silva, I., Lordello, S., Schmidt, B., \& Mietto, G. (2020). Brazilian Families Facing the COVID-19 Outbreak. Journal of Comparative Family Studies, 51(3-4). doi:10.3138/jcfs.51.3-4.008

Silva, I., Schmidt, B., Lordello, S. R., Noal, S. R., Crepaldi, M. A., \& Wagner, A. (2020b). As relações familiares diante da COVID-19: Recursos, riscos e implicações para a prática da terapia de casal e família. Pensando Famílias, 24(1), 12-28.

Teti, M., Schatz, E., \& Liebenberg, L. (2020). Methods in the Time of COVID-19: The vital role of qualitative inquiries. International Journal of Qualitative Methods, 19, 1-5. https://doi.org/10.1177/1609406920920962

World Health Organization, W. H. (WHO, 2020). WHO announces COVID-19 outbreak a pandemic. World Health Organization. Retirado de https://www.euro.who.int/en/health-topics/health-e 